\title{
IMPACT OF DISPARITY ERROR ON USER EXPERIENCE OF INTERACTING WITH STEREOSCOPIC 3D VIDEO CONTENT
}

\author{
Haiyue Yuan, Janko Ćalić, Anil Fernando, Ahmet Kondoz \\ I-Lab, Centre for Vision, Speech and Signal Processing, University of Surrey \\ h.yuan, j.calic, w.fernando,a.kondoz@surey.ac.uk
}

\begin{abstract}
The stereoscopic three-dimensional (3D) displays can offer immersive experience to the audience by artificially stimulating binocular stereopsis in the human visual system. The binocular disparity between the left and right view is the key factor in creating the impression of depth, distinguishing the stereo 3D video from other types of video paradigms. Taking into consideration of the imperfections of current disparity estimation algorithms, this paper focus on the impact of disparity error on the user experience of pointing and selecting stereo 3D content. The conducted user study into perception tolerance suggests that users can tolerate disparity errors to a certain degree, where the level of tolerance varies with perceived distance from the screen. In addition, the study demonstrates that for a typical interaction task, reduction of accuracy is proportional to the disparity level of targeted 3D objects.
\end{abstract}

Index Terms - User experience, user performance, disparity error, stereoscopic 3D video, disparity error, perception,3D interaction

\section{INTRODUCTION}

The development of 3D display technology has recently brought a whole new experience to the consumer, offering a gamut of 3D multimedia services from 3D movies in cinemas to broadcasting of 3D TV. Among the emerging 3D display technologies, stereoscopic 3D displays with the compatible $3 \mathrm{D}$ video content have been introduced to the consumer electronic devices market and have become increasingly accessible to the general public. The emergence of 3D video content has raised a lot of interest in the research community, with a considerable amount of work focusing on 3D content capture, production, and delivery. On the other hand, there has been very little research conducted focusing on meaningful user interaction with stereoscopic 3D video content. Most of the research that focuses on interaction with 3D content has been addressing only $3 \mathrm{D}$ computer generated (CG) content, which inherently offers much more information relevant to user's viewing and interaction with content. Unlike the 3D CG content, stereoscopic 3D video only provides pixel-based information of the left and right view in order to induce the illusion of depth. Therefore, the traditional 3D interaction techniques cannot be applied to stereoscopic 3D video content due to nonexistent information of the scene geometry. Having all these in mind, the main aim of our research is to study user practices, propose technical solutions and provide design guidelines to developers of interactive stereoscopic 3D video applications.

As concluded in previous work [1], the fundamental requirement for the stereoscopic 3D video interaction is selection. Based on the classification of selection process [2], the selection process consists of object indication, selection confirmation, and feedback. In order to achieve accurate selection of stereoscopic 3D video content, the accurate disparity estimation or ground truth disparity map is required. However, in case of distortion due to the effects of compression, transmission errors or absence of ground truth disparity map, the disparity estimation is critical to interaction with stereoscopic 3D video content. There is a large amount of research aimed at producing accurate disparity maps [3] from stereoscopic images. However, there is no algorithm that can produce perfect disparity map, especially in real time. We took a look at the best 3 stereo matching algorithms in terms of percentage of bad pixels and the time needed to compile the algorithm from Middlebury Stereo Matching Evaluation Database [3].

The ranking is based on the average percentage of bad pixels. The disparity error threshold is 1 , which means that the pixel refers to a bad pixel when its disparity error larger than 1 pixel. Mei et al. [4] developed an accurate stereo matching system based on absolute difference (AD) Census. For this algorithm, the average percentage of bad pixels is 3.97\%, and the time needed for implementation is 2.5 seconds, 4.5 seconds, 15 seconds, and 15 seconds for 'Tsukuba', 'Venus', 'Teddy', and 'Cones' test samples respectively. Wang and Zheng [5] presented a stereo matching algorithm based on inter-regional cooperative optimization, where the average percentage of bad pixels is $4.41 \%$ and the average time consumption is 20 seconds. Klaus, Sormann and Karner [6] developed an algorithm that utilise the color segmentation, can offer $4.23 \%$ of bad pixels with average 19 seconds to compile. These algorithms can produce relatively high quality of 
disparity map, however they are all time consuming. Therefore they are not appropriate for implementation in real time applications of interactive stereoscopic $3 \mathrm{D}$ video system. In addition, the current delivery strategy for the stereoscopic 3D video content over different network needs different compression mechanism. Compression can change the video signal, and thereby affect the disparity map generation [7]. Different algorithms for disparity estimation can produce different level of disparity error. Here we present two user studies to investigate the impact of disparity error on selecting stereoscopic 3D content. The first user study is dedicated to the issue of object indication, which focus only on impact of disparity error on user's perception tolerance. The second study investigates the impact of disparity error on the interaction task.

The reminder of this paper is organised as follows. Section 2 describes the first user study of investigating impact of disparity error on perception tolerance, followed by the second user study presented in Section 3. Section 4 of this paper highlights the main findings and provides the discussion and conclusion.

\section{IMPACT ON PERCEPTION TOLERANCE}

Object indication is the first step in the process of selection [2]. It is the stage to provide the indication of the object, which the user wants to select. The accurate indication of the targeted object provides the basis for the following accurate selection. Therefore, the object indication is a essential and fundamental part of the selection process. Having in mind of the visual distortion caused by the erroneous disparity, the accuracy of object indication for selecting stereoscopic 3D video object could be affected by the disparity error. In addition, no current algorithms can guarantee $100 \%$ accuracy of disparity estimation. Therefore, it is necessary to investigate the impact of the disparity error on the users' perception tolerance in order to have appropriate mechanism for the future design of interactive stereoscopic 3D video applications.

\subsection{Pariticipants \& Apparatus}

There were 20 participants recruited for this experiment. 18 participants were male and 2 participants were female, aged between 24 to 35 years, of which 10 were university students, 6 were university researchers, 2 participants were employed outside the university and 2 participants were unemployed. All the participants have previous experience of watching 3D stereoscopic video or movies. Before conducting the experiments, we asked participants to take a Randot stereo acuity test, and all of them had accepted stereo perception.

The experiment was performed on a 46" JVC stereoscopic display with passive polarization glasses (Model number GD463D10). The resolution of the display was $1920 \times 1080$ with recommended viewing distance of $2 \mathrm{~m}$ from the screen. The display has the width of $1.02 \mathrm{~m}$ and height of $0.57 \mathrm{~m}$. We used

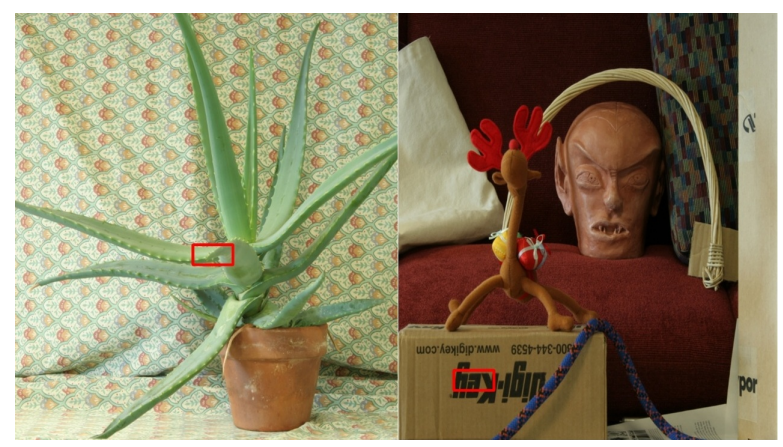

Fig. 1. Illustration of testing regions indicated in red rectangle

the mouse and keyboard as input devices. The supported format for stereoscopic content was left and right side-by-side representation. The targeted stereo $3 \mathrm{D}$ representation used in this paper addresses the interaction with post processed stereoscopic 3D content in real time but not the interactive computer graphics.

\subsection{Procedure \& Design}

There were 10 groups of images. Each group consists of a left image with its associated disparity map, and the corresponding right image. 4 groups were from Middlebury Stereo Vision page [3]. 6 groups were generated using Object Oriented Graphics Engine (OGRE) [8]. Participants were asked to gradually change the disparity of the testing regions by increasing the disparity and decreasing the disparity until they cannot tolerant the visual discomfort of viewing the testing regions. For each test set, there were 4 testing regions whose disparity was modified by participants. Each testing region has the correct disparity ranged from -10 pixels to +20 pixels. We used 10 groups of images, and each group formed a test set of the study. Therefore each participant needed to complete $2 \times 4 \times 10$ ( 2 disparity error signs, 4 testing regions, and 10 test sets) trials in total. The average time to finish all trials is 8.4 minutes, and the maximum time is just over 12 minutes. The testing regions were indicated by a red rectangle on the screen (see Figure 1). Before the experiment started, there was a opportunity for each participant to have a trial test in order to get familiar with the experiment.

\subsection{Experimental Results}

\subsubsection{Impact of the disparity error sign}

Following the data collection, the impact of disparity error sign was investigated and evaluated in relation to the perception tolerance. The perception tolerance level was indicated by the extreme values of disparity set by each participant, which refers to the highest tolerated disparity error at that depth. Larger acceptable disparity error at a given depth implies higher perception tolerance. The positive disparity error 
Table 1. Tolerance to disparity errors across the full range of perceived depth.

\begin{tabular}{|l|l|l|l|l|l|}
\hline Disparity (d) & $-10<\mathrm{d}<-5$ & $-5<\mathrm{d}<-2$ & $-2<\mathrm{d}<2$ & $2<\mathrm{d}<10$ & $10<\mathrm{d}<20$ \\
\hline Positive disparity error & 22 & 20 & 19 & 20 & 23 \\
\hline Negative disparity error & 14 & 15 & 12 & 13 & 11 \\
\hline Mean & 18 & 17.5 & 15.5 & 16.5 & 17 \\
\hline
\end{tabular}

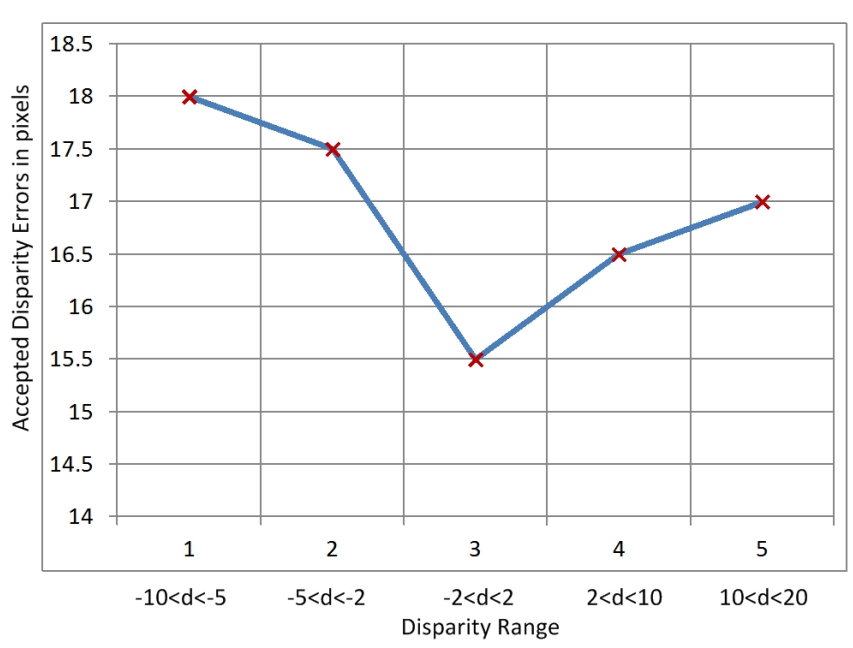

Fig. 2. Accepted average disparity error across the full range of perceived depth

represents the disparity error caused by increasing the disparity, and the negative disparity error represents the disparity error caused by decreasing the disparity. ANOVA (analysis of variance) was used to test the statistic difference between positive disparity error and negative disparity error. The results suggest that there is a significant effect of disparity error sign $(\mathrm{F} 1,1591=84.74, \mathrm{p}<0.001)$ on the perception tolerance level.

In addition, the results suggest that participants were more tolerant to positive disparity error than negative disparity error. Up to 20 pixels was acceptable level for positive disparity error, while less than 13 pixels were acceptable for negative disparity error. It suggests that the participants are more sensitive (less tolerant) to the negative disparity error than positive disparity error.

\subsubsection{Perception tolerance across the whole range of per- ceived depth}

In our experiments, each testing region had its own initial disparity ranged from -10 to 20 pixels. We divided the initial disparity into five disparity ranges (see Table 1). The aim was to investigate the perception tolerance of disparity error in relation to different disparity ranges. Table 1 describes the distribution of accepted disparity error across five disparity ranges. Similar to the findings mentioned above, the tolerance to positive disparity errors is higher than to negative disparity errors across the whole range of perceived depth.

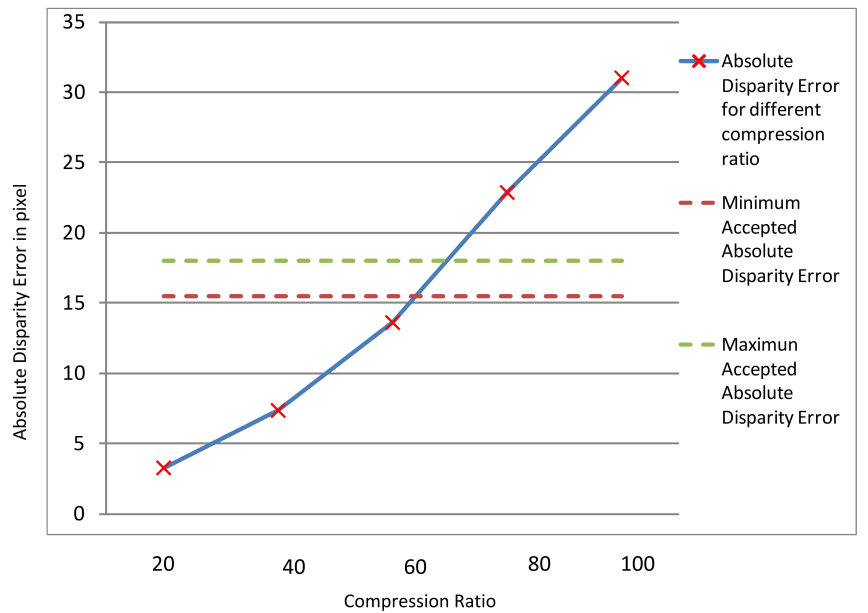

Fig. 3. Comparison of best results from [7] with the findings

Furthermore, we looked into the average accepted disparity error across the disparity range (see Figure 2). At the testing regions with low initial disparity (from -2 pixels to 2 pixels) the experiment showed lowest level of perception tolerance, which means that participants were more sensitive to disparity errors at the screen level. Nevertheless, participants still accepted disparity errors up to 15 pixels in this testing region. For testing regions further away from the screen, the level of perception tolerance was proportional to the distance from the screen level. 15 out of 1920 pixels is the minimum accepted average absolute disparity error from our experiment. As concluded in the work presented by Forster et al. [7], it describes the absolute disparity error in pixel due to different compression algorithms. The resolution of the test sequence in their work was $1176 \times 660$, and our results are based on the test sequence with resolution of $1920 \times 1080$. Therefore we translated their results equivalent to the resolution of $1920 \times 1080$.

We compared the best results from work of Forster et al. [7] with our findings. Figure 3 illustrates the comparison. The red dash line indicates the minimum accepted average absolute disparity error found from this experiment, and the green dash line indicates the maximum accepted average absolute disparity error. The blue line represents the absolute disparity error caused by using JM H.264 inter stereo compression algorithm with different compression ratio [7]. As the compression ratio stays below 60 , the absolute disparity error caused by compression is below the minimum 
Table 2. Description of disparity error range in pixels

\begin{tabular}{|l|l|l|l|l|l|}
\hline Disparity error $(\mathrm{d})$ & $\mathrm{d}<-10$ & $-10<\mathrm{d}<-5$ & $-5<\mathrm{d}<5$ & $5<\mathrm{d}<10$ & $\mathrm{~d}>10$ \\
\hline Disparity error range & 1 & 2 & 3 & 4 & 5 \\
\hline
\end{tabular}

accepted absolute disparity error found in our experiment, which means the audience might be able to tolerant the visual discomfort caused by the disparity error. As the compression ratio stays approximately between 60 to 70, the absolute disparity error caused by compression is falling between the minimum and maximum accepted absolute disparity error found in this study. Within this range, the perception tolerance depends on the starting disparity range of the selected region, which is related to the findings in section 2.3.2. In addition, as the compression ratio is over 70 , the absolute disparity error caused by compression is over the maximum accepted absolute disparity error so that the audience may not be able to bear with the discomfort due to the disparity error.

\section{IMPACT ON INTERACTION}

Selection of the stereoscopic 3D video content requires the user to point and select the video content. The first step in the selection process is the indication of the targeted object in a user interaction task. The disparity error caused by imperfection of the disparity estimation algorithm introduces distortion of the visualised video object. In the first user study, we have looked at the impact of the disparity error on the object indication. In this part, we focus on the next stage of the selection process, which is the selection confirmation and feedback. This requires visual feedback in the form of pointer that indicates the location of potential selection. The distortion of the targeted video object can potentially influence the completion of the selection task.

\subsection{Pariticipants \& Apparatus}

This study included a total number of 15 participants. All participants were male and aged from 24 to 30 years, of which 10 participants were research students from university, 3 participants were research staff and 2 participants were unemployed. This experiment had the same apparatus as the first user study. We used mouse as the input device in this experiment.

\subsection{Procedure \& Design}

The task was designed based on the ISO 9241-9 multidirectional tapping task. The participants were presented with 8 circular targets, arranged in a circle at the centre of the screen (see Figure 4). Participants were first given introduction of this experiment. Practice trials were available for participants to get familiar with the experimental task. Participants were instructed to select each highlighted target as soon and as accurately as possible using mouse. Following the ISO

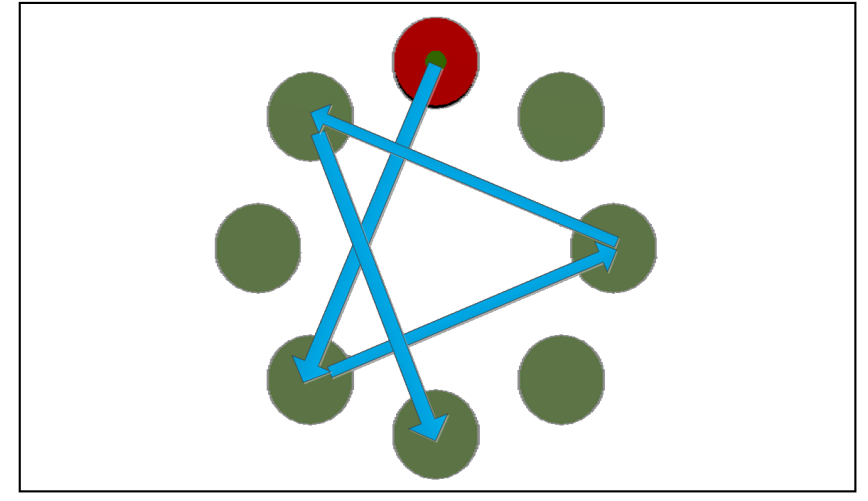

Fig. 4. Demonstration of multi-directional tapping task

9241-9 paradigm, target order started with the "top most" target and always went across the circle.

Each participant needed to use a pointer on the screen to select the targets for 8 trials. In theory the target and the pointer should have the same disparity to offer the correct depth visualisation to the viewer. As the aim of this study is to investigate the disparity error on the interaction, we manually introduced erroneous disparity to the experiments. The disparity for each target and the disparity for the pointer were randomly assigned in each trial. The difference between the the two disparities represented the disparity error.

This study used a $2 \times 3 \times 5$ within-subjects design. The independent variables were state of eye selection (one eye selection, two eyes selection), size of the pointer (half of the original, original size, and two times the original size), and disparity error ranges (see Table 2). The dependent variables were completion time and accuracy. The completion time was the time interval from selection of one target to selection of the next target. As participants did not start each trial from the same position, completion time for first target was discarded. The accuracy was indicated by the distance between the selection end point to the edge of the target divided by the radius of the target.

\subsection{Experiment Results}

Results were analysed using ANOVA and Tukey-Kramer multiple comparisons at the 5\% significance level. The statistical results are presented in Table 3. Overall we cannot find any significant effect towards the task completion time. On the contrary, the results suggest that eye selection state, pointer size, and disparity error has significant impact on task accuracy. 
Table 3. Statistical report. (Significant effects are marked $*$ for $\mathrm{p}<0.05, * *$ for $\mathrm{p}<0.01$ and $* * *$ for $\mathrm{p}<0.001$ )

\begin{tabular}{|l|l|l|}
\hline Factor & Completion time & Accuracy \\
\hline & $\mathrm{F}(\mathrm{p})$ & $\mathrm{F}(\mathrm{p})$ \\
\hline (E)ye selection & $1.21(0.27)$ & $85.66(* * *)$ \\
\hline (P)ointer size & $0.74(0.48)$ & $16.44(* * *)$ \\
\hline (D)isparity error & $1.23(0.3)$ & $29.10(* * *)$ \\
\hline E x P & $0.05(0.96)$ & $2.96(*)$ \\
\hline E x D & $0.89(0.41)$ & $1.55(0.2)$ \\
\hline P x D & $1.05(0.39)$ & $2.49(*)$ \\
\hline E x P x D & $0.97(0.38)$ & $1.42(0.24)$ \\
\hline
\end{tabular}

\subsubsection{Eye selection state}

We looked into the accuracy of the task completion in the relation to eye selection state. ANOVA analysis indicates that there is a significant difference $(\mathrm{F} 1,950=85.66, \mathrm{p}<0.001)$ of accuracy between one eye selection and two eyes selection. The average accuracy for one eye selection is $87 \%$, while for two eyes selection it is $70 \%$. In addition, significant effect at $5 \%$ level was found using the Tukey-Kramer pairwise comparisons between one eye selection and two eyes selection.

\subsubsection{Size of the pointer}

As we mentioned, the pointer had three different sizes in this experiment. ANOVA analysis indicates that there is significant difference in accuracy between different sizes of the pointer $(\mathrm{F} 2,950=16.44, \mathrm{p}<0.001)$. The original size has the highest accuracy of $80 \%$, and the double size has lowest accuracy of $69 \%$ while the half size has the accuracy of $72 \%$. The Tukey-Kramer pairwise comparisons was applied to compare the correlation within three sizes. The results revealed that the difference of task completion accuracy between half size and double size pointer is not significant. However, significant difference has been found between half size and original size, and between original size and double size pointer. In summary, using original size of pointer can achieve significantly better task completion accuracy compare with using half size and double size of pointer.

\subsubsection{Disparity error effect}

We looked into the impact of disparity error to the interaction task in terms of completion time and accuracy. The data of using one eye selection was excluded, as the disparity of pointer with one view was not available. The statistical results are depicted in Table 4.

The results suggested that the size of the pointer and the disparity error range has significant effect to the task completion accuracy, while there is no significant effect to the task completion time. The task completion accuracy of different disparity error ranges are presented in Figure 5. The partici-
Table 4. Statistical report with two eyes selection. (Significant effects are marked $*$ for $\mathrm{p}<0.05, * *$ for $\mathrm{p}<0.01$ and $* * *$ for $\mathrm{p}<0.001$ )

\begin{tabular}{|l|l|l|}
\hline Factor & Completion time & Accuracy \\
\hline & $\mathrm{F}(\mathrm{p})$ & $\mathrm{F}(\mathrm{p})$ \\
\hline (P)ointer size & $0.41(0.66)$ & $25.55(* * *)$ \\
\hline (D)isparity error & $1.10(0.36)$ & $24.23(* *)$ \\
\hline P x D & $0.97(0.46)$ & $1.39(0.2)$ \\
\hline
\end{tabular}

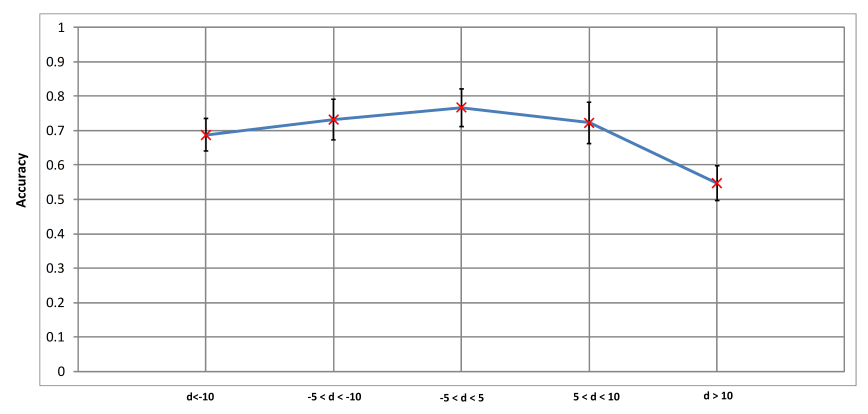

Fig. 5. The selection accuracy for different disparity error

pants had less than $70 \%$ accuracy if the disparity error is less than -10 pixels. As the disparity error is in range of -5 pixels and 5 pixels, the task completion accuracy is reaching $80 \%$. While the disparity error is greater than 10 pixels, the accuracy drops dramatically by almost $20 \%$. The sharper gradient of selection accuracy indicates that positive disparity error has more impact on the interaction task than negative. It is more difficult for participants to have accurate selection for positive disparity error than for negative.

Furthermore, the Tukey-Kramer test proves the significant differences of task completion accuracy among different disparity error ranges. In addition, we looked into the interaction effect of pointer size and disparity error, the task completion accuracy of different pointer size within different disparity error range is shown in Figure 6. The overall accuracy of the original size is higher than the half size and double size pointer across the whole range of disparity errors. However the difference of accuracy between half size and double size is not significant.

This part investigated the possible factors that can affect the user performance of completing a multi-directional tapping interaction task in a stereoscopic virtual environment. Displaying only one view of an object in the stereoscopic image to the user can produce the so-called 'ghost' effect, which is similar to present the object with transparency. Although one eye selection can help participants to make more accurate selection. However, according to the informal interview with the participants after the experiments, they stated that the 'ghost' effect is strange and not natural for viewing. In regard to pointer size, there is a significant impact on the selection accuracy. Too small or too big size of the pointer can have 


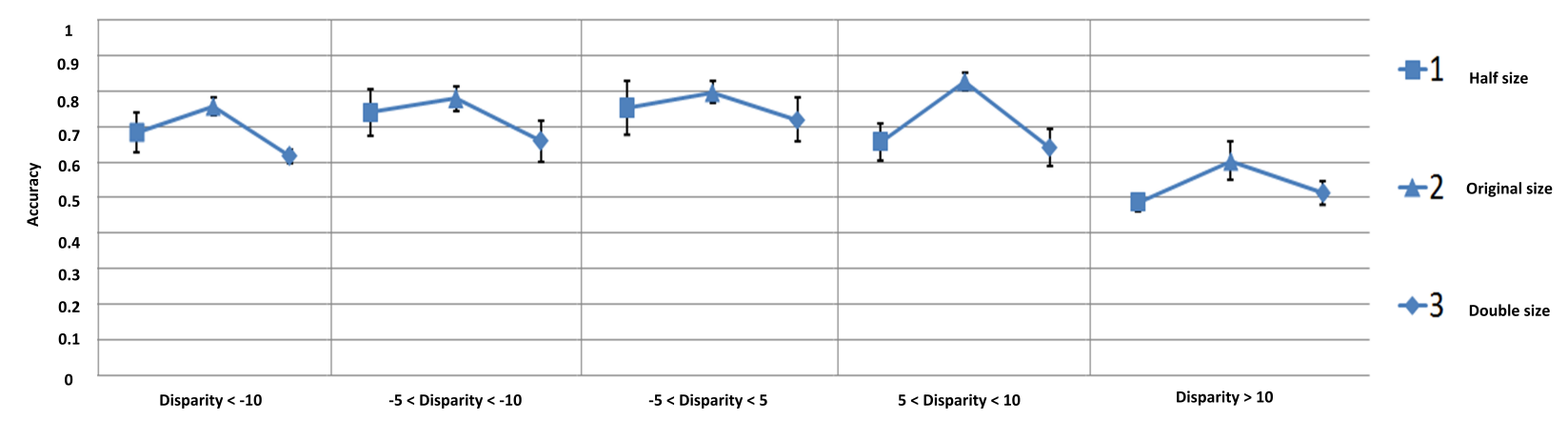

Fig. 6. Accuracy by disparity error combination, and pointer size

negative impact on the selection accuracy.

Furthermore, the investigation of disparity error indicates that different disparity errors can lead to different user performance. For selection accuracy, around the disparity error ranged from -5 to 5 pixel can provide the best user performance in terms of selection accuracy. The difference between best performance and worst performance of the selection accuracy is more $20 \%$.

\section{CONCLUSION}

This paper presents the analysis of the impact of disparity error from the perspective of interacting with stereoscopic 3D video content. The study of perception tolerance of a selected region in stereoscopic images found out that participants have certain level of tolerance to the disparity error. The results suggest that the disparity error sign has impact on perception tolerance, where participants have higher tolerance for positive disparity error than negative disparity error. Furthermore, participants have decreasing perception tolerance of disparity error towards the screen level. The study of interaction task has more complicated results with regards to impact of disparity error. For a typical multi-directional tapping interaction task, disparity error is not the only factor can affect participants' performance. Size of the pointer, and the way of presenting the pointer to participants have their impact on the user performance.

The implication of the results can be used for developing real time stereoscopic 3D video interactive application based on disparity estimation. To produce accurate disparity map can be computational expensive. However, we found that users have tolerance to disparity error, therefore an algorithm that can produce good enough disparity map but less computational cost might be more applicable in this scenario. In addition, consider the possible time consuming issue of disparity estimation, and the impact of compression/delivery to disparity estimation, we hope this study can provide understanding and guideline to design disparity estimation algorithms and compression/delivery schemes that can facilitate stereoscopic 3D video interaction.

\section{ACKNOWLEDGMENT}

This work has been supported by the MUSCADE Integrating Project (www.muscade.eu), funded under the European Commission ICT 7th Framework Programme

\section{References}

[1] Haiyue Yuan, Janko Calic, Anil Fernando, and Ahmet Kondoz, "User requirements elicitation of stereoscopic $3 \mathrm{~d}$ video interaction," in Multimedia and Expo Workshops (ICMEW), 2012 IEEE International Conference on, july 2012, pp. $31-36$.

[2] Doug A. Bowman, Chadwick A. Wingrave, Joshua M. Campbell, and Vinh Q. Ly, "Using pinch gloves for both natural and abstract interaction.," in Proc. HCI International 2001, 2001, pp. 629-633.

[3] "Middlebury stereo vision evaluation," http://vision.middlebury.edu/stereo/eval/ Accessed: 18/09/2012.

[4] Xing Mei, Xun Sun, Mingcai Zhou, Shaohui Jiao, Haitao Wang, and Xiaopeng Zhang, "On building an accurate stereo matching system on graphics hardware," in Computer Vision Workshops (ICCV Workshops), nov. 2011, pp. $467-474$.

[5] Zeng-Fu Wang and Zhi-Gang Zheng, "A region based stereo matching algorithm using cooperative optimization," in Computer Vision and Pattern Recognition, 2008. CVPR 2008, june 2008, pp. $1-8$.

[6] A. Klaus, M. Sormann, and K. Karner, "Segmentbased stereo matching using belief propagation and a selfadapting dissimilarity measure," in Pattern Recognition, 2006. ICPR 2006, 0-0 2006, vol. 3, pp. 15 -18.

[7] J. Forster, Xin Jiang, A. Terzis, and A. Rothermel, "Evaluation of compression algorithms for automotive stereo matching," in Intelligent Vehicles Symposium (IV), 2012 IEEE, june 2012, pp. 1017 -1022.

[8] "Ogre - open source graphics engine," www.ogre3d.org, Accessed: 18/09/2012. 